\title{
Le véhicule hybride rechargeable : un nouveau système sociotechnique
}

\section{Magali Pierre et Jérôme Huguet}

\section{(2) OpenEdition}

1 Journals

\section{Édition électronique}

URL : https://journals.openedition.org/artefact/7844

DOI : $10.4000 /$ artefact.7844

ISSN : 2606-9245

Éditeur:

Association Artefact. Techniques histoire et sciences humaines, Presses universitaires du Midi

\section{Édition imprimée}

Date de publication : 18 juin 2015

Pagination : 93-110

ISBN : 978-2-271-08155-1

ISSN : 2273-0753

\section{Référence électronique}

Magali Pierre et Jérôme Huguet, «Le véhicule hybride rechargeable : un nouveau système sociotechnique », Artefact [En ligne], HS 01 | 2015, mis en ligne le 30 avril 2021, consulté le 05 mai 2021. URL : http://journals.openedition.org/artefact/7844 ; DOI : https://doi.org/10.4000/artefact. 7844

\section{(c) (†) $\odot$}

Artefact, Techniques, histoire et sciences humaines est mise à disposition selon les termes de la Licence Creative Commons Attribution - Pas d'Utilisation Commerciale - Pas de Modification 4.0 International. 


\title{
Le véhicule hybride rechargeable: un nouveau système sociotechnique
}

\author{
Magali PIERRE et Jérôme HugueT*
}

\section{Résumé}

Le véhicule électrique induit-il de nouvelles façons de se mouvoir? Ce texte, qui se situe dans le champ de la sociologie des usages, présente les évolutions que le véhicule hybride rechargeable apporte dans le système de mobilité des utilisateurs. Il s'agit en effet, pour les salariés le testant pendant les trois années de l'expérimentation, de modifier leur mode de conduite, mais également d'intégrer la pratique de recharge aux habitudes quotidiennes, afin de rester le plus possible en mode électrique. Les auteurs montrent également que les modalités de mise à disposition du véhicule (voiture de fonction, de service, ou dans un pool) clivent des usages différenciés du dispositif. En effet, l'attribution d'un véhicule à titre individuel (ou non) au sein de l'entreprise est un facteur explicatif des usages, tout aussi puissant que le caractère hybride rechargeable du véhicule: cette attribution nominative encourage des usages réguliers (notamment pour les trajets domicile-travail) et autorise une charge fréquente à domicile, autant d'aspects qui accroissent l'attachement à ce véhicule. Ces modalités contrastées de mise à disposition soulignent l'importance du dispositif sociotechnique complet, autrement dit de l'articulation entre déplacements et stationnement au sein du système de mobilité des individus.

Mots-clés: sociologie des usages, système sociotechnique, véhicule électrique et hybride rechargeable.

* Formée à la sociologie et à l'anthropologie (université Paris V-René Descartes), Magali Pierre travaille depuis 2001 au sein d'EDF Recherche et Développement. Ses recherches portent depuis quelques années sur la mobilité spatiale des individus et sur l'émergence du véhicule électrique dans les sociétés occidentales. Elle assure depuis 2009 le suivi sociologique d'expérimentations déployant le véhicule électrique et l'infrastructure de recharge en France, examinant l'appropriation de cette innovation technique dans les situations d'expérimentations de grande ampleur (projets démonstrateurs).

Jérôme Huguet, titulaire d'une licence en psychologie, puis d'un master de sciences sociales (magistère de l'université Paris V-René Descartes), travaille depuis 13 ans comme sociologue consultant auprès d'organisations publiques ou privées. L'observation, les entretiens et la production universitaire sont chaque fois mobilisés pour éclairer les conditions de réception d'une offre marchande ou d'un service public. Privilégiant les approches qualitatives, nombre de ses travaux analysent ainsi la rencontre entre une offre et ses destinataires en termes de logiques d'usages, de dynamique des interactions et de contexte d'intervention. 


\section{Abstract}

Does the electric vehicle imply new ways of travelling? How does the use of electricity for fuelling the electric vehicle modify the mobility system (driving style and parking behaviors) of the actors? As a matter of fact, the electric vehicle is not only a new product but it also reorganizes the whole travelling sociotechnical system. This text, based on a classical sociology of uses, presents the evolutions that this kind of vehicle induces. Some experimentators have indeed changed their way of driving and their whole mobility system during the long term trial, in order to stay in an electric driving mode as much as possible. The plug-in-hybrid vehicle underlines this fact even more than full electric vehicles, as the electric driving mode is not mandatory but voluntarily chosen. This text also shows that the changes that we can observe are not only related to the product (the electric vehicle) but also to the conditions of its diffusion. The ways of provisioning the electric vehicles in the involved organizations structure the perception and the use of the electric vehicles. We will analyze the links between the ways of provisioning the cars (allocation to one or several persons, for professional usage only or not, etc.) and the emergence of a new mobility system.

Keywords : electric vehicle, innovation studies, plug-in-hybrid vehicle, sociotechnical system.

La communication médiatique actuelle sur le véhicule électrique, qui insiste sur la nécessité de réduire les gaz à effet de serre, met généralement en avant le produit voiture, et c'est en tant que produit que ses attraits et limites sont généralement mentionnés, notamment la question du prix à l'achat et celle de l'autonomie de la batterie. Pour autant, il s'agit là non seulement d'un nouveau produit, mais d'une reconfiguration complète $\mathrm{du}$ système sociotechnique ${ }^{1}$ - réseau d'entretien des véhicules électriques, fourniture de l'électricité pour se déplacer, installation et maintenance des infrastructures de recharge, conception de services, etc. Cet oubli du système sociotechnique dissimule probablement une difficulté à le penser et des incertitudes quant à sa configuration.

Du point de vue des usages, qu'en est-il? Comment cette question du système sociotechnique se déclinet-elle au niveau microsocial? De quelle manière l'usage de l'électricité pour se déplacer modifie-t-il (ou non) le mode de conduite ou encore le stationnement des individus? Cet article relate l'arrivée du véhicule à traction électrique dans le cadre d'une expérimentation ${ }^{2}$ dont l'objectif fut de valider un certain nombre de points techniques et commerciaux, ce qui a permis d'apporter des réponses à ces questions. Le cas du véhicule hybride rechargeable (VHR) présente en effet la question du changement avec encore plus d'évidence, puisque le recours au mode électrique n'est pas obligatoire. Notre analyse vise en outre à montrer en quoi le véhicule à traction électrique est une innovation qui réactive l'importance du système sociotechnique dans toutes ses dimensions. 
Cette réflexion sur la genèse de cadres d'usage de la voiture à traction électrique situe notre article à la fois dans le champ de la sociologie des usages et de la sociologie des techniques et de l'innovation. Ainsi, en analysant l'appropriation d'un dispositif sociotechnique par des utilisateurs dont les perceptions et les usages sont pris en compte dans un dispositif de retour d'expérience, nous nous situons principalement dans le champ de la sociologie des usages et de la consommation, dans le sens où «la sociologie des usages [...] [étudie] l'usage resitué dans l'action sociale ${ }^{3}{ }$.

\section{Contexte général et caractéristiques de l'expérimentation}

Notre analyse fait suite à une enquête de terrain menée dans le Bas-Rhin, enquête dont le recueil de données a été réalisé en plusieurs étapes, au cours des années 2010-2011, auprès du public expérimentant des véhicules hybrides rechargeables (VHR) et l'infrastructure de charge, ainsi qu'auprès des gestionnaires de flottes et d'autres acteurs du projet.

Strasbourg est une collectivité locale pionnière et volontariste en matière de transports en commun, de restriction de l'accès au centre-ville par les voitures et de mobilité régionale. Cependant, dans cette aire urbaine, la croissance démographique (ménages et emplois) est portée par le péri-urbain. Le Bas-Rhin est ainsi une zone de forte mobilité - 3,9 déplacements par jour et par personne, dont $55 \%$ dans un rayon de moins de $3 \mathrm{~km}$, d'après l'Enquête Ménages Déplacements 2009 -, mais la voiture y représente une grande part: elle couvre la moitié des déplacements dans la zone de Strasbourg et plus des deux tiers dans les autres zones du Bas-Rhin (régions de Saverne, Piémont-Vosges, Alsace du Nord, Sélestat).
L'expérimentation dont nous rendons compte ici repose sur un partenariat avec des organisations de tous types: un consortium est à l'initiative du projet (entre un fournisseur d'énergie, un constructeur automobile et une collectivité locale, sous l'égide de l'ADEME); les partenaires testant le dispositif sont des organisations nombreuses (une trentaine) et de tous types (établissements publics et collectivités territoriales, segments locaux de grandes entreprises, PME, etc.).

Cette expérimentation a consisté à mettre à disposition des partenaires, établissements et entreprises du BasRhin environ quatre-vingts VHR, ainsi que l'infrastructure de charge permettant de les alimenter en électricité. Une quinzaine de points de charge publics ont été placés en voirie et en parkings souterrains. Afin que tous les utilisateurs puissent brancher le véhicule hybride rechargeable aussi souvent que possible, l'installation de l'infrastructure de charge a eu lieu dans les espaces privatifs: au domicile des personnes bénéficiant d'une voiture attribuée à titre individuel (voiture de fonction ou 
de service) et sur les principaux sites des partenaires concernés ${ }^{4}$.

Choisis par les entreprises partenaires, les individus éligibles à participer à l'expérimentation devaient couvrir des distances annuelles assez élevées à titre professionnel ${ }^{5}$ et disposer d'un emplacement privé et couvert de stationnement à domicile (pour ce qui est des utilisateurs disposant d'un coffret de charge à leur domicile).

Ces personnes habitent principalement en première couronne de Strasbourg et vivent bien souvent dans des pavillons. L'échantillon d'utilisateurs correspond à quatre types de profils socioprofessionnels: des patrons de PME, des techniciens travaillant sur des domaines techniques (réseaux, BTP, eau, énergie), des cadres dirigeants de grands groupes et des commerciaux, ainsi que des professionnels du transport (mécaniciens, chauffeurslivreurs). S'y ajoutent des gestionnaires de flottes de véhicules, que nous avons interrogés en tant que relais de l'expérimentation. Probablement pour des raisons tenant aux critères de sélection du public classique des véhicules d'entreprises, il s'agit donc principalement d'hommes, ayant déjà un véhicule d'entreprise et habitant dans le péri-urbain.

Dans l'expérimentation étudiée, le véhicule hybride rechargeable est présenté comme une innovation: on peut brancher ce véhicule pour recharger sa batterie, mais il conserve un moteur à explosion qui accroît sa capacité de rayonnement au-delà de ce qu'autorise classiquement une voiture électrique ${ }^{6}$. Ainsi, à la différence de la voiture conventionnelle comme de la voiture électrique, le véhicule hybride rechargeable qui puise son énergie sur le réservoir d'essence ou sur la batterie électrique, se refuse à la détermination technique: il laisse ouvert, pour chacun, la possibilité de favoriser ou non le mode électrique en adoptant une conduite adéquate (trajets courts, charge fréquente, éco-conduite, etc. ${ }^{7}$.

Le véhicule hybride rechargeable, moyen terme entre voiture à essence et voiture électrique, pose à ce titre constamment question aux utilisateurs, qui peuvent infléchir son fonctionnement (mode électrique versus hybride) en fonction de leur manière de conduire. La qualification de ses propriétés reste donc une question ouverte pour les utilisateurs - on parlera de qualités du produit plutôt que de caractéristiques, parce que «parler de qualités, c'est poser la question du processus, controversé, de qualification ${ }^{8} »$. Le rapport au système sociotechnique, c'est donc le rapport au véhicule, mais aussi à l'infrastructure de charge. Il redonne donc une place importante au stationnement, puisque les moments stationnaires correspondent aux moments où le véhicule est ré-alimenté en électricité.

Ce véhicule, dont la justification tient pour une grande part à la question de la limitation des émissions de carbone, propose par ailleurs un ensemble d'afficheurs permettant de mettre en œuvre une gestion des consommations d'essence: le tableau de bord affiche une jauge électronique indiquant le niveau de la batterie électrique, une représentation de la circulation des énergies dans le véhicule (de la batterie vers les roues ou l'inverse en mode freinage récupératif), ou encore des graphes synthétisant les performances de la batterie sur les derniers trajets. Les outils d'aide à la gestion des consommations qui sont proposés dans le véhicule font donc un lien 
entre les deux aspects (le véhicule et l'infrastructure de charge), car ils rendent compte des consommations d'énergie du véhicule.

\section{Méthodologie d'enquête}

Les VHR et les points de charge ont été livrés progressivement entre avril et novembre 2010, à partir de la date d'inauguration officielle. Nous avons rencontré une quarantaine de personnes en entretiens semi-directifs, à leur domicile ou à leur entreprise, dans leur véhicule ou dans leur bureau. Les techniques qualitatives (enquête de terrain) choisies pour cette enquête ont permis de rendre compte de la logique d'action des utilisateurs et de rentrer dans la complexité des pratiques. Plus précisément, nous nous sommes entretenus avec vingt utilisateurs à trois reprises; compte tenu que ces utilisateurs pouvaient rapporter le véhicule chez eux et en avaient donc un usage fréquent, nous les nommons utilisateurs réguliers; à quoi, nous avons ajouté des entretiens avec une vingtaine d'utilisateurs occasionnels et acteurs du projet (principalement des gestionnaires de flottes de véhicules d'entreprise), mais seulement lors des deuxième et troisième phases d'enquête (une fois le dispositif technique livré - T2, et un an plus tard - T3).

La première phase d'enquête (T1), constituant une phase témoin visant à mieux connaître le public, à comprendre les déplacements et à percevoir le rapport à leur actuelle voiture d'entreprise, a été réalisée au cours du premier semestre 2010 (entre mi-février et mi-avril), c'està-dire avant que les utilisateurs pilotes $\mathrm{du}$ dispositif ne reçoivent le véhicule hybride rechargeable et les coffrets de charge. Des observations ont également pu être menées, quelques entretiens ayant été suivis d'une visite du futur emplacement de stationnement au domicile, et d'une visualisation rapide de l'emplacement de stationnement au travail. Par ailleurs, nous rendons également compte de certains propos tenus lors des cinq réunions-utilisateurs auxquelles nous avons participé, ces réunions servant à expliquer le détail du projet aux utilisateurs volontaires pour l'expérimentation'.

La deuxième phase d'enquête (T2), dont le recueil de données d'entretiens a été réalisé à l'été 2010 (entre avril et octobre 2010), a eu lieu quelques mois après le lancement officiel du projet (fin avril 2010), c'est-à-dire après une courte période d'utilisation du dispositif (deux mois après la livraison du véhicule), et a donné lieu à des observations de terrain portant sur les comportements au volant et sur les pratiques de charge.

Pour la troisième phase d'enquête (T3), le recueil de données a eu lieu à l'été 2011, autrement dit dans une période où les habitudes sont acquises et les routines prises. Quelles sont en effet les pratiques de mobilité et de recharge du véhicule, plus d'un an et demi après «la première fois»? Les résultats restituent donc des éléments issus de la phase de mise en route (T2) et confortés dans la suite de l'expérimentation (T3). Le suivi au long 
cours permet d'éclairer tout particulièrement les changements qui se sont fait jour dans le système de mobilité des personnes.

\section{Des changements importants de l'ensemble du système de mobilité}

Quelles sont les pratiques de déplacement, de stationnement et de charge depuis que les utilisateurs bénéficient du dispositif sociotechnique? Autrement dit, le système de mobilité des expérimentateurs est-il inchangé ou observe$\mathrm{t}$-on des modifications au cours de cette phase de découverte, en termes de trajets, de façons de conduire, de manière de stationner, voire d'appréhender ses consommations d'électricité? Plus encore, à quoi tiennent les changements constatés?

\section{L'aspect environnemental, au cœur de l'engagement}

Quels sont les intérêts du système hybride selon les utilisateurs? Les participants à l'expérimentation ont une perception globalement très positive du projet et de sa portée. La plupart du temps, les avantages mentionnés sont reliés à des enjeux globaux, alors que les inconvénients sont souvent $\mathrm{d}^{\prime} u n$ registre personnel (perte de confort, etc.). Ainsi, le principal intérêt du dispositif est lié aux enjeux environnementaux et ce thème se décline un peu différemment selon les individus, voire pour un même individu, selon que la question environnementale est renvoyée sur les aspects $\mathrm{CO}_{2}$, pollution locale ou encore environnement sonore.
Un véhicule produisant peu de $\mathrm{CO}_{2^{\prime}}$ voilà l'intérêt le plus souvent cité des motorisations électriques, d'après les personnes interrogées. Les faibles émissions de gaz à effet de serre renvoient à des enjeux de préservation de l'environnement au niveau planétaire.
Alexandra: «On parle de plus en plus de réchauffement de la planète, de chan- gement climatique et de soucis d'envi- ronnement, donc c'est obligé de faire quelque chose. Je pense que ça doit être bien perçu par tout le monde.» (T2)

Le faible niveau de pollutions locales émises par le VHR permet de limiter la pollution locale. Ainsi, le VHR devient un véhicule urbain idéal, car, en ville, c'est surtout le moteur électrique qui fonctionne.

Marc: «Je pense que le principal avantage, qu'il soit électrique ou hybride rechargeable, c'est sa propreté en utilisation, en particulier urbaine. En général, puisqu'il y a des moyens de recharge, je pense que favoriser l'utilisation de l'énergie, la production nucléaire de nuit, etc., va être extrêmement intéressante pour gagner en indépendance énergétique. Lié donc à la propreté et de manière générale...» / Intervieweur: «Oui. Et quand vous parlez de pro- 
preté, c'est plutôt le $\mathrm{CO}_{2}$ ou les autres pollutions?» / Marc: «C'est l'ensemble. Qu'elles soient sonores, ce qui est un peu secondaire parce qu'on a quand même fait de grands progrès, mais surtout l'ensemble de la pollution, y compris celle $\mathrm{qu}^{\prime}$ on ressent, bon, le $\mathrm{CO}_{2^{\prime}}$ mais l'ensemble des particules émises clairement dans un progrès indéniable.» (T2)

Pour certaines personnes participant à l'expérimentation, la bonne performance environnementale du véhicule fait écho à des valeurs écologiques avec lesquelles elles sont personnellement en concordance $^{10}$.

\section{Des changements appréciés dans la conduite et le stationnement}

Les consommations d'essence des VHR sont liées aux fréquences de charge: pour un même trajet, plus souvent on recharge le véhicule, moins on consomme d'essence. Aussi note-t-on, dans les discours, une bonne volonté généralisée: tout le monde veut se charger le plus possible, car c'est ce qui conditionne la possibilité de pouvoir rouler en mode électrique.

Pierre: «Je crois que l'atout du VHR, c'est quand vous faites des circuits urbains ou de domicile-bureau comme je le fais, c'est quand même satisfaisant de savoir qu'on peut rouler à l'électricité sur ces trajets-là sans polluer et puis sans risque de toute façon même de tomber en panne.» (T2)

La conduite "détendue» est liée au fait de rester le plus possible sur le mode électrique. Elle est source d'un certain plaisir. Comme pris au jeu, les conducteurs font en sorte de «doser leur accélération» afin de rester en mode électrique.

Claude: «Je dose l'accélération. [...] J'accélère plus doucement ou, si je vois que ça monte trop vite, je ralentis tout de suite pour que ça rebaisse et après, je réaccélère plus doucement. » (T3)

La plupart des utilisateurs soulignent la détente que favorise l'absence de bruit. Le silence est une qualité appréciée pour la majorité des personnes et participe d'un environnement de conduite qui modifie le vécu de la voiture.

Rémi: «Sur la manière de conduire, oui clairement, je roule plus relax, plus zen, moins vite.» / Enquêteur: "Moins vite qu'avec une Smart?» / Rémi: «Oui.»/ Enquêteur: «Et ça, vous l'attribuez au mode électrique, au fait qu'il y ait du silence...?» / Rémi: «Je peux parler du côté ludique. C'est marrant de dire: "Tiens, j'ai de l'électricité, autant rester en électrique". Au lieu de faire du 120, je fais du 90 ou 100. Je ne suis pas plus en retard pour autant et puis au moins, j'ai fait du transport doux. Après, je pense que oui, inconsciemment, quand on roule sans vibration, sans bruit, j'imagine que l'inconscient fait qu'on recherche plutôt ce type d'ambiance.» (T2)

Conduire est devenu une activité agréable où il devient possible de discuter au téléphone, d'écouter de la musique ou de profiter du silence. Ce qui participe à se détendre au volant, et par là même, de conduire «cool». 
Chloé: «Je me suis remis des CD dans la voiture alors que je n'écoutais plus de CD dans la voiture, parce que... En gros, j'ai le sentiment d'être moins nerveuse, je ne sais pas si c'est le bon terme, mais d'être moins speed au volant. C'est une conduite plus souple, plus cool.» (T2)

Un signe de cet engouement pour le mode électrique: l'émulation est assez forte au sein des partenaires, dont certains proposent spontanément que s'organise un concours de la plus faible consommation d'essence.

Didier: "Ce qui serait intéressant, c'est qu'à travers les différents partenaires, on fasse un ranking, comment dire, un classement... Qui c'est qui arrive à économiser le plus ou qui exploite au maximum la partie électrique, ça, c'est intéressant. » (T2)

Ainsi, si les trajets n'ont pas changé, en revanche le mode de conduite s'est fortement adapté pour la plupart d'entre eux: plus souple pour ménager l'autonomie de la batterie, plus attentif à l'environnement car l'absence de bruit oblige le conducteur à être vigilant aux piétons.

Ce changement dans la façon de conduire est apprécié, notamment car les utilisateurs ont le sentiment que la voiture hybride rechargeable met à leur disposition les outils permettant d'assumer leurs efforts, et partant de les alléger. L'attention portée au mode de conduite (moins nerveuse, plus anticipatoire, plus à l'écoute de l'environnement physique) prend en effet appui sur les outils de gestion des consommations qui sont mis à la disposition des utilisateurs dans leur véhicule.
Florent: «J'utilise la petite réglette [du tableau de bord]: j'essaie de ne pas taper dans la pédale et puis, je tâche de rester en tout électrique, tant qu'à faire.» (T2)

Comme l'indique Madeleine Akrich, le dispositif technique sous-entend une certaine définition de l'actant; quand l'actant qui se saisit de l'objet n'a pas les compétences requises, il doit se coordonner pour les acquérir (accommodements, arrangements locaux): cela nécessite sa coopération ${ }^{11}$. De manière générale, les utilisateurs n'ont pas modifié leurs déplacements, mais certains ayant identifié et noté les paramètres influant sur les performances de consommation, ils ont tendance à faire en sorte de rouler sur le mode le plus favorable pour garder l'autonomie de leur batterie. Parmi ces paramètres on compte: le poids, la vitesse et les à-coups dans le rythme, le vent, le froid.

Laurent: "C'est une curiosité aussi de [...] se tester sur la volonté que l'on a de recourir à cette possibilité-là. Par exemple, je me suis déjà posé la question de savoir si j'étais prêt à rentrer en modérant ma vitesse et en ayant recours à l'énergie électrique, plutôt que de rentrer en utilisant toutes les possibilités de l'infrastructure autoroutière qui me permet de rouler à $130 \mathrm{~km} / \mathrm{h}$ et qui, du coup, fait appel plutôt à l'énergie thermique.» (T2)

Tout cela plaide pour appuyer la thèse $\mathrm{du}$ changement de régime sociotechnique, du point de vue des utilisateurs: la conduite comme le stationnement et l'état d'esprit du conducteur se trouvent modifiés par l'expérience 
de conduite d'un véhicule hybride rechargeable.

\section{Un dispositif qui nécessite la coopération des actants}

L'utilisateur «idéal» coopère entièrement avec le dispositif. Cependant, d'autres utilisateurs se refusent à la nécessaire coopération entre l'utilisateur et le dispositif. Par exemple, ceux qui sont en retrait de toute logique d'implication ne sont pas prêts à engager la coopération minimale à l'utilisation du dispositif technique. Ainsi, un des utilisateurs ne recharge jamais le véhicule car le cordon lui semble peu ergonomique. Quelques personnes ont ainsi tendance à considérer l'infrastructure de charge comme de "[simples] prises de courant». Ils considèrent donc les services et fonctionnalités secondaires des bornes (communication, identification de l'utilisateur, rapatriement de données, sécurisation de la charge, etc.) comme nuisant à la fonction primordiale de livraison d'une électricité de qualité.

Le référentiel des utilisateurs réalisant de longs trajets et se chargeant peu souvent reste alors la voiture conventionnelle (puisqu'ils utilisent ainsi le véhicule hybride rechargeable sur un mode conventionnel) - l'un d'entre eux parle ainsi du mode hybride comme d'un mode «normal». Ces utilisateurs, jugeant le véhicule au regard de voitures thermiques, ne se satisfont pas de ses consommations. Notamment, ils aimeraient disposer d'une batterie d'une plus grande autonomie $(50 \mathrm{~km})$ compte tenu de leurs besoins spécifiques, voire d'un réservoir d'essence plus important aussi, de manière à faire le plein moins souvent.
Yann: «[70/30], c'est déjà pas mal mais si on avait une autonomie un poil plus importante, de l'ordre de 50 kilomètres, je suis quasiment persuadé que je serais à 50/50 [en mode électrique/hybride].» (T2)

Dominique: "Autre élément un peu désagréable, dont je me suis aperçu en faisant le plein la première fois, c'est que la capacité $\mathrm{du}$ réservoir est très faible. C'est 35 litres. C'est très faible. Pour moi qui fais quand même des trajets assez importants, c'est moyen.» (T2)

Ceux qui ne changent pas de référentiel de conduite ont tendance à reprocher au VHR son manque de puissance, qui impose selon eux une cadence trop lente (donc stressante); et ils sont déçus de la boîte automatique et se lassent de la charge fréquente.

Didier: "Je préfère les sportives, qui ont de la reprise.» (T3)

Leur représentation commune est de souligner que la vie professionnelle impose un rythme qui précisément ne permet pas d'être «cool» au volant, que ce soit lors des mouvements pendulaires où «ce n'est pas possible de ralentir la circulation le matin», ou lors de grands trajets.

D'autres, plus nombreux, semblent relever les «qualités» du VHR, entendues comme des spécificités appréciées: l'éco-conduite, la conduite silencieuse, qui permet de se détendre et est considérée sur le registre du confort. Ces derniers ajustent leur usage aux possibilités techniques du véhicule. Ils apprécient le fait que cette voiture soit automatique et 
pratiquent davantage la conduite «cool» grâce à la boite de vitesses automatique.

Chloé: «J'ai le sentiment d'être moins nerveuse, je ne sais pas si c'est le bon terme, mais d'être moins speed au volant. C'est une conduite plus souple. Ça, c'est lié à la boîte auto. Je pense qu'avec n'importe quelle boîte automatique, on a une conduite plus fluide.» (T3)

Enfin, certains sont véritablement convertis au véhicule électrique, grâce à l'expérimentation. Ils considèrent le VHR comme réduisant les contraintes liées à la conduite automobile et ont du plaisir à conduire un véhicule électrique. Le plaisir qui y est associé comporte un aspect ludique. Ces personnes sont celles qui se centrent davantage sur les supports de gestion des consommations d'énergie pour en saluer les vertus en tant qu'aide à l'éco-conduite.

Olivier: "J'essaie de comprendre un peu l'algorithme de la voiture, d'en jouer et de l'optimiser [...]. Et je [le] retrouve, moi, quand je prends d'autres véhicules. Je suis influencé. Quand je prends mon scooter, je roule vachement plus cool maintenant. » (T3)

Ainsi, ils se distinguent également des autres par le fait de considérer que le référentiel «cool» est désormais celui dominant, qui fonctionne également quand ils utilisent une autre voiture, alors que les précédents soulignaient davantage le basculement qu'ils opéraient en retrouvant une voiture ordinaire.

Laurent: "Je pense que ce sont des habitudes que l'on prend et qu'on ne laisse pas forcément dehors quand on reprend son véhicule personnel. » (T3)

Ainsi se dessinent les liens entre registre de préoccupation privilégié et type de conduite qui ensemble construisent une représentation générale sur cette voiture différente. Quelques-uns n'apprécient guère cette voiture, pratiquent peu l'éco-conduite et centrent leur attention sur la puissance de la voiture, qui fait défaut. Pour les autres, l'usage de cette voiture a d'abord été l'occasion de découvrir avec plaisir le confort de conduite que procurent les voitures automatiques, en s'allégeant de la contrainte de devoir passer les vitesses.

S'impliquer dans le projet nécessite que l'on ait pris connaissance du fonctionnement du dispositif, en suivant les réunions à ce sujet, en lisant la documentation fournie, en remplissant son dossier de candidature, etc. (présentation en interne, connaissance des enjeux et des délais de livraison du dispositif, relations antérieures avec les partenaires, etc.). Mais, outre ces connaissances préalables pour lesquelles l'ensemble des participants arrivés à ce stade se sont montrés curieux $^{12}$, la manière dont on s'implique dans le projet trouve notamment son origine dans la capacité décisionnaire du bénéficiaire: est-on à l'initiative du projet ou le «subit»-on? Certains utilisateurs sont en effet des cadres dirigeants ou des chefs d'entreprise et sont à l'initiative de la participation de leur établissement à l'expérimentation.

Au sein des départements élus, le choix des utilisateurs finaux (qui aura le droit d'usage des VHR) relève d'une décision hiérarchique, qui se veut valorisante. En effet, il ne s'agit pas de n'importe quelle 
voiture: ce n'est pas une voiture «de technicien» mais une «berline de luxe» (expression de Ronald, T3).

Luc: «Le choix au niveau [de mon entreprise] a été fait globalement au niveau du comité de direction. Je pense qu'un point important a été l'aspect $\mathrm{RH}$ globalement, l'aspect avantage en nature.» (T3)

Des critères variables selon les éta- blissements président ainsi aux choix d'affectation finaux. Ainsi, selon que la hiérarchie est, au sein de ces départements, très formelle ou plutôt souple, selon les besoins au sein de chacun des départements (calendrier de remplacement des voitures de fonction), selon la motivation de la hiérarchie intermédiaire, etc., les VHR se voient ainsi attribués à des utilisateurs permanents (donc attribution nominative) ou à des utilisateurs occasionnels (en flotte).

\section{L'attribution des véhicules dans les entreprises, un facteur clivant}

Tous les participants à l'expérimentation parcourent un nombre assez important de kilomètres par mois $(2000 \mathrm{~km}$ en moyenne d'après leurs déclarations) et partagent leurs déplacements entre des trajets locaux et des trajets sur de longues distances, que seuls les utilisateurs de voitures de service attribuées personnellement semblent peu nombreux à accomplir.

L'attribution d'un véhicule est un des outils des politiques internes d'entreprise. C'est donc au terme de décisions managériales que les VHR se voient attribués aux collaborateurs remplissant les conditions nécessaires (kilomètres parcourus, configuration domestique, etc.). En effet, les modalités de mise à disposition des véhicules commandent différents cadres d'usage possibles ${ }^{13}$. Ainsi, les différentes directions des éta- blissements concernés décident d'affecter les voitures d'entreprise sous trois modalités: au titre de voitures de fonction (c'est-à-dire pour des besoins professionnels et personnels), au titre de voitures de service attribuées personnellement ou encore au titre de voitures mutualisées au sein d'un "pool» de véhicules.

Dans les deux premiers cas, le véhicule a été attribué personnellement, et il s'agit d'utilisateurs nominatifs du véhicule, qui disposent alors d'un coffret de charge au domicile; les utilisateurs empruntant le véhicule auprès d'un pool peuvent être considérés comme des utilisateurs occasionnels (voir tabl. 1). En somme les modalités d'attribution du véhicule clivent des publics, sous l'angle $\mathrm{du}$ degré d'implication dans l'expérimentation. 


\begin{tabular}{|c|c|c|c|}
\hline $\begin{array}{l}\text { Nombre } \\
\text { d'utilisateurs }\end{array}$ & $\begin{array}{l}\text { Statut du } \\
\text { véhicule }\end{array}$ & Trajets réalisés & $\begin{array}{l}\text { Présence ou non de } \\
\text { coffret domestique }\end{array}$ \\
\hline \multirow{3}{*}{$\begin{array}{l}\text { Un seul = } \\
\text { Utilisateur régulier } \\
\text { (voiture attribuée } \\
\text { personnellement } \\
\text { ou nominative) }\end{array}$} & $\begin{array}{l}\text { Voiture de } \\
\text { fonction }\end{array}$ & $\begin{array}{l}\text { Trajets professionnels } \\
+ \text { Domicile-travail } \\
+ \text { Privés }\end{array}$ & $\begin{array}{l}\text { Coffret de charge } \\
\text { domestique }\end{array}$ \\
\hline & $\begin{array}{l}\text { Voiture de } \\
\text { service }\end{array}$ & $\begin{array}{l}\text { Trajets professionnels } \\
+ \text { Domicile-travail }\end{array}$ & $\begin{array}{l}\text { Coffret de charge } \\
\text { domestique }\end{array}$ \\
\hline & & $\begin{array}{l}\text { Trajets professionnels } \\
\text { (rare) }\end{array}$ & $\begin{array}{l}\text { Pas de coffret } \\
\text { domestique (rare) }\end{array}$ \\
\hline $\begin{array}{l}\text { Des utilisateurs } \\
\text { occasionnels } \\
\text { (voiture partagée) }\end{array}$ & $\begin{array}{l}\text { Voiture de } \\
\text { service } \\
\text { mutualisé }\end{array}$ & $\begin{array}{l}\text { Trajets professionnels } \\
\text { seulement }\end{array}$ & $\begin{array}{l}\text { Pas de coffret } \\
\text { domestique }\end{array}$ \\
\hline
\end{tabular}

\section{Les modalités différencient des publics, sous l'angle des distances parcourues et de la fréquence de charge}

Ces modalités de mise à disposition des voitures (voiture de fonction, voiture de service attribuée personnellement, ou voiture de pool) correspondent à des prescriptions d'utilisation: la voiture sert-elle à se déplacer pour des besoins seulement professionnels ou des besoins aussi personnels? Si les VHR de fonction sont davantage utilisés sur de longues distances, cela provient de la possibilité d'usage dans un cadre privé, notamment les départs en vacances.

Enquêtrice: «À combien de kilomètres vous êtes à peu près?»/
Alexandra: «En tout? Je crois 27000 en un peu plus d'un an. [...] En général, elle fait par jour à peu près [...] 25-30 km par jour en courses ou pour le courrier. [...] [Elle roule beaucoup] en vacances ou alors quand je vais voir des clients parce qu'on a des clients dans toute la France.» (T3)

Ces modalités de mise à disposition engagent donc également à des potentialités différenciées ${ }^{14}$. Ces éléments sont très structurants, car ils commandent à la fois la fréquence à laquelle on va pouvoir se servir du véhicule (longueur des trajets parcourus) et la manière dont on va pouvoir le recharger. En effet, un usage "électrique» de ce véhicule, à la différence d'une voiture conventionnelle, nécessite qu'il soit branché tous les 
$20 \mathrm{~km}$, si bien que les modalités de mise à disposition conditionnent le fait de pouvoir en avoir un usage optimal (en rechargeant chez soi) ou non - sachant qu'il y a consensus sur cet optimum parmi les participants.

De manière générale, dans la plupart des établissements concernés, l'emprunt d'un VHR en pool (flotte de véhicules partagés), sans être complexe, n'est pas direct: demande d'obtention formalisée, centralisation des clés, etc. Ainsi, ces modalités de mise à disposition du véhicule sont directement corrélées à une plus ou moins grande facilité d'emprunt du véhicule. Plus précisément, pour les utilisateurs occasionnels (autrement dit ceux qui utilisent la voiture au sein d'un pool), les modalités d'emprunt sont souvent complexes: faire une réservation, obtenir un accord, aller au bureau où se trouvent les clés, se déclarer et signer, prendre la voiture et se réhabituer au mode de conduite, etc. Ainsi, la complexité de l'attribution du VHR pour les utilisateurs de pool explique un recours relativement limité.

Clément: "La clé [de la Prius] est encore devant, dans le bâtiment. Donc [si j'avais voulu l'emprunter aujourd'hui], il aurait fallu aller [jusqu'au bâtiment qui est] devant, monter et chercher la clé et redescendre.» / Florent: «C'est compliqué.» / Enquêteur: «Vous redéposez la clé à chaque fois? / Clément: "Oui. [...]»/ Florent: «Il y a les papiers et tout ça qui sont également...» / Florent: «On les partage, en fait, avec le patron [qui est aussi un utilisateur de la Prius]. Elles sont chez lui par principe. C'est normal.» (T2)
De ce fait, les utilisateurs occasionnels se voient souvent attribuer la VHR pour des trajets exceptionnels, c'est-à-dire longs. Ils se retrouvent donc dans une situation d'usage où le mode électrique leur est inaccessible.

Jean: «Là, il me l'a donnée sur un gros trajet dans le sens où $[. .$.$] c'était$ le seul véhicule qui était disponible sur la date et sur les journées correspondantes.» (T2)

En somme, les modalités de mise à disposition des voitures rendent compte des types de mobilité qui se font jour. Ainsi, les utilisateurs de voitures de fonction sont ceux qui parcourent les distances les plus longues avec leur VHR, les utilisateurs de voitures de service attribuées personnellement étant à l'autre bout de l'axe. Pour ce qui est des fréquences d'utilisation et de recharge, il nous faut opposer les utilisateurs nominatifs (voitures de fonction et de service) et les utilisateurs occasionnels, ces derniers utilisant et rechargeant moins souvent le véhicule. Ces deux axes (fréquence de charge et distances parcourues) rendent ainsi compte $d u$ "ratio électrique» du véhicule: parmi les utilisateurs réguliers, ceux qui se déplacent sur de longues distances ont des ratios électriques modérés; ceux qui font des déplacements fréquents sur de courtes distances ont au contraire une consommation très faible d'essence. Ce constat se confirme en phase de routine (T3), et nous l'avons synthétisé dans le graphe qui suit. 


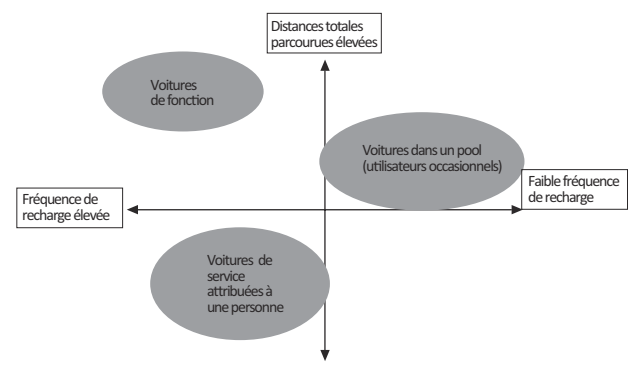

\section{Les modalités différencient des publics, sous l'angle du recours au VHR : un choix?}

Certains salariés apprécient la mise en visibilité offerte par le véhicule, et notamment ceux qui ont à porter la politique de développement durable de leur organisation d'appartenance.

Laurent: «À travers [ces efforts de 106 charge constitue une contrainte pour la gestion du parc; ou encore pour les chauffeurs d'un établissement, le VHR signifie une transformation de leur métier, car c'est une voiture bien souvent conduite par l'utilisateur désireux de se montrer au volant, donc peu appréciée par les chauffeurs destitués.

Finalement, pour les utilisateurs, la question du choix de recourir au VHR comme véhicule ne se pose pas dans les mêmes termes selon les modalités d'attribution du véhicule: le recours au VHR n'est pas un choix pour tous.

- Pour les utilisateurs de voitures de fonction: même si c'est le principal mode de transport, le VHR s'inscrit au sein d'un véritable choix modal, parmi d'autres modes de transport. Les utilisateurs de VHR de fonction se distinguent des autres par la possibilité d'utiliser leur voiture professionnelle dans la vie privée. Utilisent-ils cette possibilité? Pour la moitié d'entre eux, le VHR est leur voiture principale, y compris pour les vacances. Toujours est-il que dans le cadre familial, la notion de choix apparait entre la voiture personnelle au sein $\mathrm{du}$ foyer et la voiture professionnelle pour toute la famille. Les personnes arbitrent alors au sein de leur famille selon un comparatif entre les deux voitures.

Pour les utilisateurs de voitures de fonction, presque tous cadres supérieurs ou dirigeants, le recours au VHR prend alors place dans un système de mobilité plurimodale. En hyper centre, ils vont recourir au tramway ou au métro et, sur de plus longues distances (plusieurs centaines de kilomètres), au train ou à l'avion. Les utilisateurs de voitures de fonction ont le sentiment de gestionnaires de flottes, qui doivent gér des voitures dans lesquelles la durée de 
choisir le VHR au sein d'un portefeuille modal.

- Pour les utilisateurs de voitures de service attribuées personnellement: le véhicule sert pour les trajets domicile-travail, outre les trajets professionnels. Cette possibilité est généralement très appréciée.

- Les utilisateurs en pool n'ont pas toujours le sentiment d'un choix: souvent l'attribution est liée à la disponibilité.

La question du choix est peu présente pour les utilisateurs occasionnels, puisqu'il s'agit plus souvent d'une attribution en fonction des voitures disponibles au moment voulu - mais elle est remplacée par la question de l'attribution. Cette attribution sélective, outre le fait qu'elle va à l'encontre du choix, peut conduire à un effet pervers: pour limiter le nombre d'utilisateurs, on est prêt à prêter le VHR à des utilisateurs s'en servant régulièrement pour des distances longues, donc sur un régime moins performant.

Le fait que le VHR soit un véhicule partagé amène en outre à une série d'étapes (qui sont routinières chez les utilisateurs réguliers de VHR) à se rejouer à chaque fois: se former à l'utilisation, emprunter le véhicule, etc. L'itinéraire d'usage du VHR se révèle donc plus complexe pour les utilisateurs au sein d'un pool.

\section{Les modalités différencient des publics, sous l'angle de la recharge du véhicule}

La norme, nous l'avons $\mathrm{vu}$, consiste à recharger le plus possible le VHR. La charge au travail est l'occasion principale de recharge, pour l'ensemble des utilisateurs. La fréquence de charge y est assez élevée (de 1 à 3 charges déclarées par jour), et notamment le matin en arrivant sur son site professionnel ou après un déplacement (de retour de la pause méridienne ou après un rendez-vous). Cette fréquence de recharge reste élevée tout au long de l'expérimentation, ce qui est bien un indice de la qualité de l'engagement des utilisateurs et de leur nette préférence pour le mode électrique.

Mais, pour les utilisateurs occasionnels, lors de la phase de mise en route, les pratiques de charge oscillent entre délégation aux gestionnaires de flotte et défection (ne pas se charger du tout). En cas de délégation de la charge au gestionnaire de flotte, la charge dépend alors de la disponibilité du délégataire. Cette attribution, qui peut donner aux utilisateurs le sentiment d'un dévoiement du véhicule par rapport aux attentes portées par l'expérimentation, correspond sinon à un échec, du moins à un «détachement» de la part d'une bonne partie des utilisateurs occasionnels ${ }^{15}$. Le dispositif $\mathrm{d}^{\prime}$ intéressement ${ }^{16}$ a ainsi failli, puisque la charge leur est presque impossible et conséquemment le mode électrique inconnu. Le caractère inapproprié du véhicule pour leurs usages rejaillit donc directement sur l'appréciation qui est portée sur les performances du véhicule en général.

Vincent: «Moi, si je devais l'acheter à titre personnel, je ne ferais pas l'investissement parce qu'il y a ce défaut qui est pour moi conséquent. Alors, si je l'ai pour aller faire les courses et que je peux la brancher toutes les 5 minutes, ça va. Mais si c'est une voiture avec laquelle, je dois me déplacer, ça n'a aucun intérêt 
pour moi, parce que j'ai fait 1200 kilomètres, je suis revenu, les batteries étaient vides.» (T2)

À noter, une évolution des modalités d'attribution des véhicules en pool par les gestionnaires de flotte permettant une meilleure gestion de la charge: certains véhicules de flotte sont devenus nominatifs et le nombre d'utilisateurs s'est restreint entre le T2 et le T3. Il s'agit là d'un effet de sélection fonctionnelle (par les gestionnaires de flotte): le public s'est restreint à quelques habitués qui connaissent bien le véhicule et le rechargent eux-mêmes (effet d'apprentissage vertueux).

Ainsi, dans une certaine mesure, la fréquence de charge varie selon les modalités de mise à disposition des voitures.

\section{Au final, des pratiques clivées par le dispositif technique}

Dans le système de mobilité qui se met en place, la nécessité de recharger le véhicule renforce l'articulation entre pratiques de déplacement et pratiques de stationnement. Le VHR est une innovation qui, bien que très plastique, réaffirme l'importance du dispositif sociotechnique (et non plus simple produit voiture) et du système de mobilité (et non plus seulement des déplacements mais aussi le stationnement).

Bien plus, les modalités de mise à disposition du véhicule, qui commandent la manière dont le véhicule pourra être utilisé (pour quels trajets? avec quelle fréquence de charge?) sont très structurantes pour comprendre le nouveau système de mobilité des utilisateurs du dispositif. En effet, les besoins de mobilité (distances parcourues et fréquence d'utilisation) correspondant aux modalités de mise à disposition des voitures influent certes sur la possibilité de rouler en mode électrique et par conséquent commandent les performances techniques du véhicule. Mais, dans le même temps, les modalités de mise à disposition donnent ou retirent un sentiment de connaissance technique et de maîtrise des capacités du véhicule, nécessaire à l'expression de jugements de valence positive. Pour le dire vite, le VHR est davantage apprécié par ceux qui s'en servent souvent sur des circuits courts, autrement dit ceux qui ont le sentiment de choisir leur mode de déplacement, ce qui est lié aux modalités de mise à disposition du véhicule.

Bien plus, alors que les utilisateurs réguliers du véhicule se trouvent relativement satisfaits, les utilisateurs occasionnels sont plus critiques face à un véhicule qu'ils trouvent parfois inadapté à leurs usages. En effet, ces derniers se voient affecter le véhicule pour des trajets de longue distance, après une réservation complexe, et n'ont pas la main sur la procédure de recharge. Au contraire, les utilisateurs réguliers, qu'il s'agisse de voitures de fonction ou de service, disposent $\mathrm{du}$ véhicule de manière très simple et le rechargent par eux-mêmes, 
autrement dit aussi souvent qu'ils le veulent et, s'ils le souhaitent, à leur domicile.

En somme, les modalités de mise à disposition des voitures différencient les utilisateurs réguliers et les occasionnels, sous l'angle de l'implication et du degré de connaissance du fonctionnement $\mathrm{du}$ dispositif, sous l'angle des facilités d'emprunt du véhicule, des longueurs de trajets parcourus, des capacités de recharge et du point de vue à l'égard du dispositif. À ce titre, le VHR, soulignant l'importance de l'ensemble du système sociotechnique, établit des liens entre des domaines (travail-domicile, déplacement-stationnement, etc.) trop souvent dissociés ${ }^{17}$.

Le VHR fait cohabiter deux technologies et cette partition aboutit à tracer des usages différenciés, que nous avons éclairés sous l'angle des modalités de mise à disposition des véhicules. Cet article ne prétend pas résoudre la question du changement de régime sociotechnique telle qu'elle est posée par la littérature sur la transition énergétique ${ }^{18}$. Il montre cependant que le VHR est une innovation qui réactive le clivage entre des usages que la voiture conventionnelle ne séparait pas si distinctement et qui pourraient correspondre à des objets techniques différents, dans un contexte de prise de conscience écologique.

\section{Notes}

1. B. van Bree, P. J. Verbong, C. J. Kramer, «A MLP on the intro of hydrogen and battery-electric vehicles ", Technological forecasting and social change, 77, 2010, p. 529-540.

2. Nous remercions l'ensemble des collectivités locales et entreprises partenaires et, plus particulièrement, Toyota et EDF, pour le soutien qu'elles ont apporté à cette enquête de terrain.
3. Josiane Jouet, «Retour critique sur la sociologie des usages», Réseaux, vol. 18, n 100, 2000, p. 487-521, p. 499; voir aussi Madeleine Akrich et Cécile Meadel, «Problématiser la question des usages", Sciences sociales et santé, vol. 22, $\mathrm{n}^{\circ} 1$, 2004, p. 5-20.

4. Plus exactement, pendant les trois années de l'expérimentation, plus de cent cinquante points de charge alimentent les VHR, prioritairement au domicile et sur le lieu d'emploi des bénéficiaires, mais aussi dans l'espace public (bornes en voirie et en parkings publics).

5. Entre 10000 et $45000 \mathrm{~km}$ par an, d'après les informations récoltées auprès des particuliers en entretiens.

6. Concrètement, le véhicule testé permet, en conditions réelles moyennes et si l'allure de conduite est souple, de parcourir $20 \mathrm{~km}$ en mode électrique; après quoi, le moteur bascule automatiquement sur le mode hybride, c'est-à-dire fonctionnant de manière optimisée avec de l'essence. Le constructeur automobile parle de mode hybride et non pas thermique, car, quand la voiture est sur cette position, l'énergie de freinage est récupérée pour régénérer marginalement la batterie électrique.

7. Une analyse approfondie de cette question de l'indétermination technique peut être trouvée dans Magali Pierre, "Script technique et prescriptions organisationnelles. Utiliser un véhicule hybride rechargeable en milieu professionnel», dans Jérôme Cihuelo, Catherine Granclement, Alain Jobert, Énergie et transformations sociales. Enquêtes sur les interfaces énergétiques, éditions Lavoisier, 2015, Paris; voir aussi Reid R. Heffner, Kenneth S. Kurani, Thomas S. Turrentine, Symbolism in early markets for hybrid electric vehicles, Institute of transportation studies, UC Davis, 2010.

8. Michel Callon, Cécile Méadel, Vololona Rabehisoa, «L'économie des qualités», Politix, vol. $13, \mathrm{n}^{\circ} 52,2000$, p. 211-239, p. 219.

9. Travaillant à la direction Recherche et Développement d'une des entreprises de l'équipeprojet, j'ai assisté, en parallèle de l'enquête, aux réunions de communication à destination des utilisateurs et à certaines réunions techniques.

10. Magali Pierre, «Quand préoccupations environnementales et enjeux professionnels s'entrecroisent: le cas des entreprises participant aux essais de voitures électriques », dans Patricia Lejoux et Nathalie Ortar, SHS Web of conférences, vol. 9, 2014, numéro thématique La transition énergétique: vrais enjeux, faux départs?

11. Madeleine Akrich. «Les objets techniques et leurs utilisateurs, de la conception à l'action ", Raisons pratiques, $\mathrm{n}^{\circ} 4$, 1993, p. 35-57, p. 54. 
12. Notons que la présente typologie des logiques d'implication dans l'expérimentation concerne avant tout les utilisateurs réguliers, qu'ils utilisent le véhicule comme voiture de service ou de fonction. Certains utilisateurs occasionnels manifestent des logiques d'implication identiques aux utilisateurs réguliers et on les a alors intégrés à l'analyse qui suit. D'autres, au contraire, ne sont pas vraiment impliqués dans l'expérimentation mais plutôt en retrait, et notamment ceux qui sont dans une maîtrise partielle du dispositif (à cause de dysfonctionnements dans les modalités de mise à disposition du dispositif sociotechnique) et ceux pour qui l'arrivée du véhicule suscite un conflit d'intérêt.

13. Sur le rôle des expérimentations dans la définition de cadres d'usage, Patrice Flichy, "Technique, usage et représentations», Réseaux, $n^{\circ} 148-149,2008$, p. 147-174.

14. Concrètement, ce véhicule permet-il alors d'agrandir des capacités d'action, d'être un bureau mobile, etc.?, voir Reinhard Gressel et Christophe Mundunteguy, «Des acteurs souvent ignorés de l'espace urbain, les Professionnels Mobiles», communication aux Journées "Ville, Transport et Territoire, Quoi de neuf?» du Pôle Ville de l'université Paris Est, 2010, p. 161.
15. À noter, les étapes ultérieures de l'enquête montrent que les gestionnaires de flotte ont cherché, quelques mois plus tard, à corriger cet effet pervers en modifiant les conditions d'attribution des véhicules partagés (trajets plus courts, cercle plus restreint d'utilisateurs, etc.).

16. Sur le concept d'intéressement, Madeleine Akrich, Michel Callon, Bruno Latour. «À quoi tient le succès des innovations? 1. L'art de l'intéressement», Gérer et comprendre, Annales des Mines, $\mathrm{n}^{\circ} 11,1998$, p. 4-17.

17. Tom Hargreaves, «Practice-ing behaviour change: applying social practice theory to proenvironmental behaviour change", Journal of consumer culture, 2001, p. 11-79.

18. Voir notamment Franck W. Geels, «Technological transitions as evolutionary reconfiguration processes: a multi-level perspective and a casestudy», Research policy, n 31, 2002, p. 1257-1274, ainsi que Ronald Kline et Trevor Pinch, «Users as agents of technological change: the social construction of the automobile in the rural United States", Technology and culture, vol. 37, n 4, 1996, p. 763-795. 